\title{
Propensity score analysis of 18-FDG PET/CT-enhanced staging in patients undergoing surgery for esophageal cancer
}

\author{
N. Patel ${ }^{1} \cdot$ Kieran G. Foley $^{2}$ (D) A. G. Powell ${ }^{2} \cdot$ J. R. Wheat ${ }^{1} \cdot$ D. Chan ${ }^{1} \cdot$ P. Fielding $^{3} \cdot$ S. A. Roberts $^{4} \cdot$ W. G. Lewis $^{1}$
}

Received: 16 April 2018 / Accepted: 30 July 2018 / Published online: 16 August 2018

(C) The Author(s) 2018

\begin{abstract}
Purpose PET/CT is now integral to the staging pathway for potentially curable esophageal cancer (EC), primarily to identify distant metastases undetected by computed tomography. The aim of this study was to analyze the effect of PET/CT introduction on survival and assess patterns of recurrence after esophagectomy.

Methods A longitudinal cohort of EC patients staged between 1998 and 2016 were considered for inclusion. After co-variate adjustment using propensity scoring, a cohort of 496 patients (273 pre-PET/CT and 223 post-PET/CT) who underwent esophagectomy [median age 63 years (31-80), 395 males, 425 adenocarcinomas, 71 squamous cell carcinomas, 325 neoadjuvant therapy] were included. The primary outcome measure was overall survival (OS) based on intention to treat.

Results Three-year OS pre-PET/CT was $42.5 \%$ compared with $57.8 \%$ post-PET/CT (Chi ${ }^{2} 6.571$, df $\left.1, p=0.004\right)$. On multivariable analysis, pT stage (HR 1.496 [95\% CI 1.28-1.75], $p<0.0001$ ), pN stage (HR 1.114 [95\% CI 1.04-1.19], $p=0.001$ ) and $\mathrm{PET} / \mathrm{CT}$ staging (HR 0.688 [95\% CI 0.53-0.89] $p=0.004$ ) were independently associated with OS. Recurrent cancer was observed in 125 patients (51.4\%) pre-PET/CT, compared with 74 patients post-PET/CT (37.8\%, $p=0.004)$, and was less likely to be distant recurrence after PET/CT introduction (39.5 vs. 27.0\%, $p=0.006)$.

Conclusions Enhanced PET/CT staging is an important modality and independent factor associated with improved survival in patients undergoing esophagectomy for cancer.
\end{abstract}

Keywords Esophagus $\cdot$ Neoplasms $\cdot$ Positron emission tomography $\cdot$ Survival $\cdot$ Recurrence

\section{Introduction}

Imaging is fundamental to improved cancer staging and largely guides treatment decision-making. Radiological staging

N. Patel and K. G. Foley are joint first authors.

Kieran G. Foley

foleykg@cardiff.ac.uk

1 Department of General Surgery, University Hospital of Wales, Cardiff CF14 4XW, UK

2 Division of Cancer \& Genetics, Cardiff University School of Medicine, Heath Park, Cardiff CF14 4XN, UK

3 Wales Research \& Diagnostic Positron Emission Tomography Imaging Centre (PETIC), UHW, Cardiff CF14 4XN, UK

4 Department of Radiology, University Hospital of Wales, Cardiff CF14 4XW, UK investigations including computed tomography (CT) and positron emission tomography (PET) provide anatomical and functional information and have greatly impacted clinical practice [1-3].

Positron emission tomography integrated with computed tomography $(\mathrm{PET} / \mathrm{CT})$ is now an established and evidencebased part of the modern radiological staging algorithm of esophageal cancer (EC) $[4,5]$. Reported benefits include the detection of distant metastases not detected by CT, which changes management in up to $38 \%$ of patients [6].

Yet PET/CT is not without limitations. Endoscopic ultrasound (EUS) has been reported to be superior in staging both the primary tumor and local lymph nodes [7], and no evidence has yet emerged that use of PET/CT has been associated with improved overall survival (OS) [8]. Moreover, important reconfiguration of UK esophagogastric cancer services has occurred over the last decade, which has been accompanied by better clinical outcomes. The National Oesophago-Gastric Cancer Audit (NOGCA) has emphasized that better patient selection by improved radiological and physiological staging 
accuracy, more use of neo-adjuvant chemotherapy, less postoperative morbidity and mortality, and centralization of services are all significant factors in improving survival after potentially curative esophagectomy [9]. Determining the particular role PET/CT may have played in influencing outcome after potentially curative esophageal cancer surgery is consequently challenging, and likely not to undergo a randomized control trial process.

Propensity score (PS) analysis facilitates measurement of the probability of receiving a particular treatment modality related to a number of variables, and represents a strong and powerful alternative strategy to explore cause, compared with orthodox statistical adjustment [10]. PS analysis adjusts for potential confounders, balancing important co-variables such as baseline characteristics or therapies, and is an alternative to control trials where ethical or practical challenges prohibit randomization, enhancing fair comparison [11, 12].

The aim of this study was to analyze the influence of 18FDG PET/CT introduction on OS after esophagectomy for cancer when compared with historical controls by means of PS analysis. The hypothesis was that PET/CT introduction into the routine staging algorithm of patients diagnosed with EC was associated with improved OS after potentially curative surgery. The setting was a UK Upper Gastrointestinal (UGI) cancer network serving a population of 1.8 million.

\section{Materials and methods}

All patients diagnosed with EC of any cell type who underwent surgery and had PET/CT imaging during the preoperative staging period in the South East Wales regional UGI cancer network were studied prospectively between January 1, 2009 and August 31,2016. These patients were compared with a historical cohort of consecutive patients undergoing EC surgery between January 1, 1998 and January 1, 2009, staged with the network's historical staging algorithm pre-PET/CT. Exclusion criteria included patients undergoing PET/CT for Siewert type III esophagogastric junctional cancer with proximal esophageal extension, and patients undergoing salvage esophagectomy following initial definitive chemoradiotherapy (dCRT).

Patients proceeded to PET/CT staging only if they were suitable for potentially curative treatment on the grounds of CT stage and performance status, and was concurrently arranged with EUS examination. PET/CT was used for pretreatment staging only (Fig. 1) and was not used for restaging after neoadjuvant therapy. All PET studies were integrated PET/CT and no patients received PET imaging alone. Detail of the networks' EUS staging protocol has been described previously [13]. Patients' fitness was assessed by cardiopulmonary exercise testing (CPX) [14], and the final management plan was determined at the regional cancer network

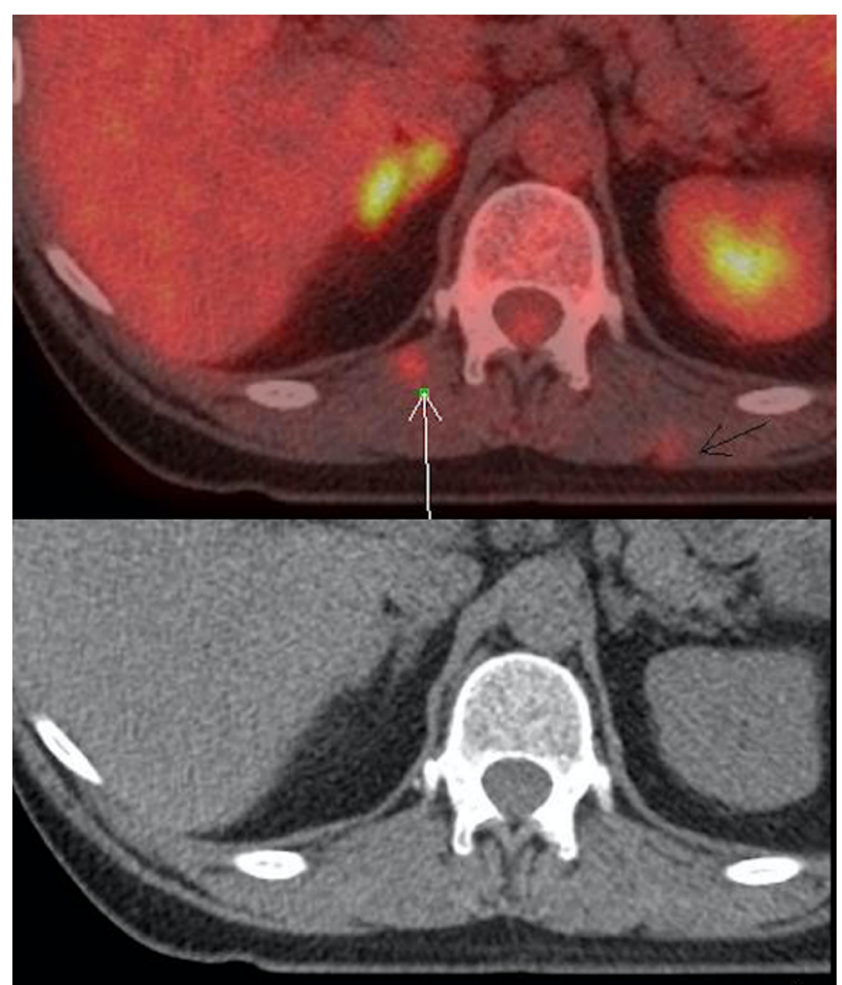

Fig. 1 Fused PET/CT (above) and CT (below) images of soft tissue metastases (white and black arrows) in a patient initially considered suitable for curative treatment. However, the management became palliative once the metastases were detected

multi-disciplinary team (MDT) meeting. All staging investigations were reported in accordance with the UICC Tumour Node Metastasis (TNM) staging methods [15]. The primary outcome measure was OS from diagnosis. Secondary outcome measures were proven recurrence patterns and diseasefree survival (DFS). Ethical approval, sought from the regional ethics committee, was waived because the study was deemed to represent service evaluation. A number of developments in the management of EC occurred during the study period, including changes in practice based upon the publication of randomized clinical trials, the introduction of an enhanced recovery program in 2008 [14] and finally, centralization of the Upper Gastrointestinal (UGI) cancer regional network service in South East Wales from August 1, 2010 [16].

\section{PET/CT protocol}

PET/CT examinations were performed at two centers. At the first center (site 1), a total of 87 patients had PET/CT examinations performed using a Philips 16 slice Gemini GXL dedicated PET/CT scanner (Philips Medical Systems, Cleveland, $\mathrm{OH}, \mathrm{USA}$ ). The uptake time was $60 \mathrm{~min}$. A standard administered activity of $350 \mathrm{MBq}$ of FDG was given. Reconstructions were performed using a 3D acquisition with non-time of flight acquisition for $4 \mathrm{~min}$ per bed position. 
Four hundred and eighty-five patients were imaged at the second center (site 2) using a GE Discovery 690 PET/CT scanner (GE Healthcare, Pollards Wood, Buckinghamshire, UK). Serum glucose levels were routinely checked and confirmed to be less than $7.0 \mathrm{mmol} / 1$ prior to imaging. Patients received an activity of $4 \mathrm{MBq}$ of ${ }^{18} \mathrm{~F}$-FDG per kilogram of body weight. Uptake time was $90 \mathrm{~min}$. PET images were acquired at $3 \mathrm{~min}$ per field of view. The length of the axial field of view was $15.7 \mathrm{~cm}$. Images were reconstructed with the ordered subset expectation maximization algorithm, with 24 subsets and two iterations. Matrix size was $256 \times 256$ pixels, using the VUE Point TM time of flight algorithm. CT images were acquired in a helical acquisition with a pitch of 0.98 and a tube rotation speed of $0.5 \mathrm{~s}$. Tube output was $120 \mathrm{kVp}$ with output modulation between 20 and $200 \mathrm{~mA}$. Matrix size for the CT acquisition was $512 \times$ 512 pixels with a $50-\mathrm{cm}$ field of view. At both centers, all patients were starved for a minimum of $6 \mathrm{~h}$ prior to imaging and no oral or intravenous contrast was administered.

The assignment of lymph nodes as involved or uninvolved on PET images at the two centers was based on subjective clinical assessment in each case. In general, nodes were only assigned as involved if they showed discernible tracer uptake above that of background and were identified separately from the primary tumor. Other factors affecting the classification of nodes were the morphology, size, and relative uptake of the node in question in comparison to the primary tumor. No specific SUV or size cut off was used in assignment of nodes as benign or malignant. Lymph nodes considered physiological or related to an alternative etiology were excluded from the $\mathrm{N}$ stage.

\section{Treatment}

Patients were selected for radical treatment (surgery or dCRT) based on perceived radiologic stage, comorbidity, and patient choice according to algorithms described previously [17-19]. The standard surgical approach consisted of subtotal trans-thoracic esophagectomy (TTO) as described by Lewis and Tanner. Trans-hiatal esophagectomy (THO), as described by Orringer, was used selectively in patients with adenocarcinoma of the lower third of the esophagus with significant cardiorespiratory risk profiles, or T1/2 N0 disease. All procedures used an open approach, and esophageal resection was defined as potentially curative if all visible tumor was removed, and both proximal and distal resection margins were free of tumor on histological examination. $\mathrm{R} 1$ resection was defined as positive longitudinal or circumferential margin status on histological examination [20].

\section{Follow-up evaluation}

Patients were reviewed every 3 months for the first year, and 6 monthly thereafter until 5 years or death. Patients underwent clinical assessment and venous blood sampling including carcinoembryonic antigen (CEA) measurements. Patients who were suspected to have disease recurrence based on clinical assessment or a raised venous CEA underwent computed tomography or endoscopy. Patterns of recurrence were defined as loco-regional, distant (metastatic), or both locoregional and distant, when both were diagnosed concurrently. The time of recurrence was taken as the date of the confirmatory investigation. The patient cohort was analyzed in January 2017. No patients were lost to follow-up and death certification was obtained from the Office for National Statistics via Cancer Network Information System Cymru (CaNISC).

\section{Statistical methods}

Grouped data were expressed as median (range) and nonparametric methods used throughout. Propensity scores were generated using appropriate logistic regression model, and included all relevant independent variables thought to be potential confounding factors and those which affect outcome. Co-variate adjustment was performed using the propensity scores, which were assessed for balance across groups using a Student's $t$ test and defined by $|\mathrm{d}|>0.25$. The variables were considered by the MDT and comprised age group, gender, tumor histology, pathological $\mathrm{T}$ and $\mathrm{N}$ stage, operation type, and site of PET/CT examination. The dependent variable was whether the patient underwent PET/CT staging. The probabilities option was chosen to generate propensity scores, which were then used in a regression, covariate adjustment, to estimate the effect of exposure of PET/CT on DFS and OS. DFS was calculated by measuring the interval from a landmark time of 6 months after diagnosis to the date of recurrence; an approach mirroring previous randomized trials [21, 22] and allowing for variance in time to definitive surgery. Events resulting in a failure to complete curative treatment, such as palliative surgery, operative mortality, and disease progression during neoadjuvant therapy, were assumed to occur at this landmark time, to facilitate intention-to-treat analysis. OS was measured from the date of diagnosis, and cumulative survival calculated according to the method of Kaplan and Meier. Differences between groups were analyzed with the log rank test. Univariable analyses examining factors influencing survival were examined initially by the life table method of Kaplan and Meier, and variables significant at the $p$ $<0.010$ level were entered into a forward conditional Cox proportional hazards model. All statistical analysis was performed with SPSS $®$ (IBM® SPSS $®$ Statistics v23.0.0.0, IBM Corporation, Armonk, NY, USA). 
Fig. 2 CONSORT diagram detailing the creation of both prePET and post-PET cohorts

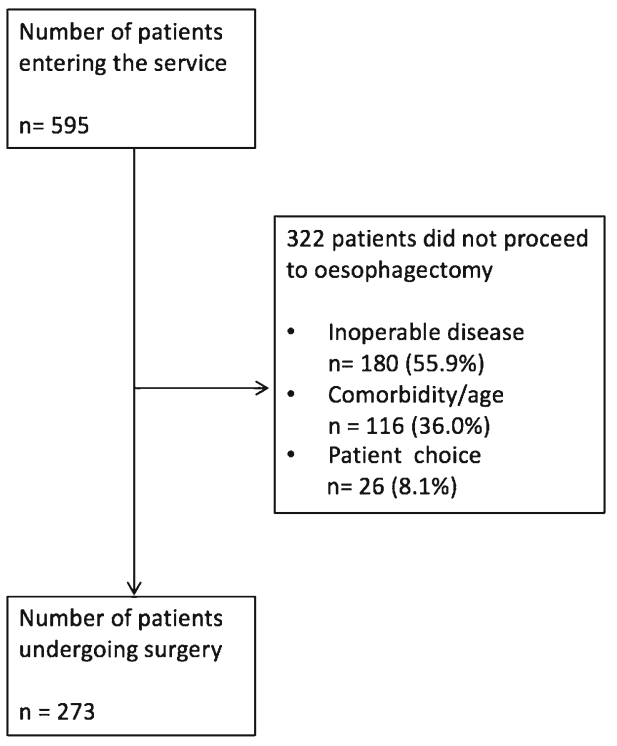

$2009-2016$

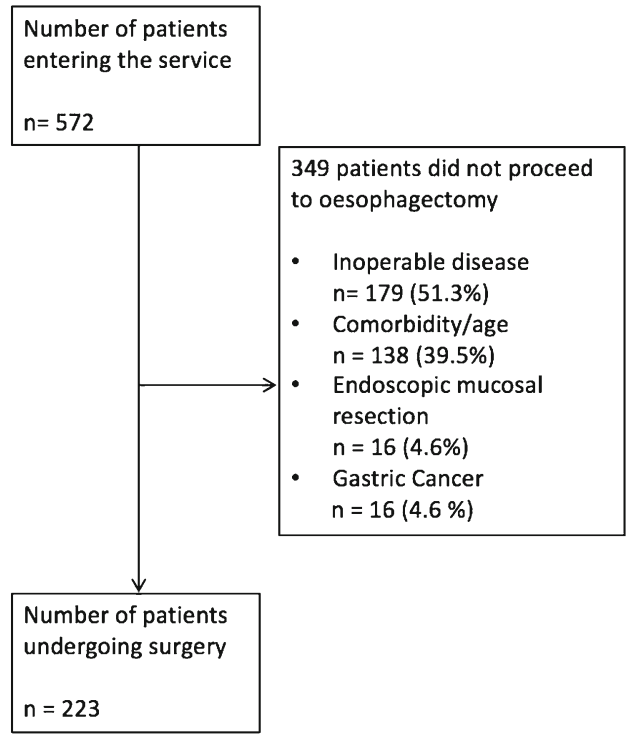

\section{Results}

In total, 1167 patients were diagnosed with EC between 1998 and 2016 (Fig. 2). Of these, 595 were diagnosed between 1998 and 2009 (pre-PET cohort) and 572 were diagnosed between 2009 and 2016 (post-PET cohort). Of the 595 in the pre-PET cohort, $322(54.1 \%)$ did not proceed to resection because 180 (55.9\%) had inoperable disease, 116 (36.0\%) had pre-existing comorbidity, and $26(8.1 \%)$ because of patient choice. Following the introduction of PET/CT (2009-2016), 572 patients were diagnosed with esophageal and junctional cancers of which $223(40.0 \%)$ underwent EC resection. Of the 349 $(61.0 \%)$ patients that did not progress to surgical resection, 179 (51.3\%) had inoperable disease and 138 (39.5\%) had preexisting co-morbidity precluding curative treatment. Sixteen (4.6\%) had predominantly gastric cancer extending above the esophagogastric junction (an exclusion criterion in this study), and $16(4.6 \%)$ underwent endoscopic mucosal resection (EMR).

Following propensity scoring, 496 consecutive patients were eligible for inclusion, 273 pre-PET/CT, and 223 patients post-PET/CT. Twenty-two (9.9\%) patients had non-avid tumors on PET/CT, but were included on an intention-to-treat analysis basis. Propensity scores were generated for all 496 patients in the cohort. The propensity scores were balanced across the two groups, Student's $t$ test $p=0.212$ and there was no covariate exhibiting a large imbalance $(|\mathrm{d}|>0.25)$. Patient demographics are detailed in Table 1.

The operative approach was trans-thoracic in 233 (47.0\%), trans-hiatal in 200 (40.3\%), and a three-stage approach was used in six $(1.2 \%)$ patients. Open and close laparotomy was performed in $57(11.5 \%)$ patients either due to un-resectable tumor or metastatic disease. Pathological examination revealed R1 resection specimens (microscopic margin involvement) in 166 (33.5\%) patients. Operative mortality occurred in 17 (3.4\%) patients within 30 days of surgery. More trans-hiatal esophagectomies and threestage esophagogastrectomies were performed in the post-PET/CT cohort than the pre-PET/CT cohort, which contained more transthoracic esophagectomies $(p<0.0001)$. The post-PET/CT cohort also underwent marginally more open and close laparotomies for undetected distant metastases $(n=27,12.1 \%)$ compared with the pre-PET/CT cohort $(n=30,11 \%, p=0.698)$. The median followup was 26 months (range, 6 to 220), with 476 patients (96.1\%) followed up for 1 year or until death, 447 (90.0\%) for 2 years, 418 $(84.3 \%)$ for 3 years, and 375 (81.4\%) for 5 years.

\section{Duration of survival}

\section{Disease-free survival}

For patients with all pathological stages of disease, median DFS was 16 months in the pre-PET/CT cohort, compared with 35 months in the post-PET/CT cohort ( $p=0.049$; Fig. 3). One-, $2-$, and 3-year cumulative DFS in the pre-PET/CT cohort was 56.5, 41.4, and $34.4 \%$ respectively, compared with $61.9,52.6$, and $49.3 \%$, in the post-PET/CT cohort. Following PET/CT introduction, median-, 1-, 2-, and 3-year DFS increased by 19 months, 5.4, 11.2, and $14.9 \%$, respectively. All factors associated with DFS on univariable analysis are shown in Table 2. 
Table 1 Details of the patients before and after PET/CT introduction

\begin{tabular}{|c|c|c|c|}
\hline & Pre-PET/CT & Post-PET/CT & $p$ value \\
\hline Number (\%) & $273(55.0)$ & $223(45.0)$ & \\
\hline Median age years (range) & $61(31-80)$ & $64(36-77)$ & $0.036 \S$ \\
\hline$<50$ & $38(13.9)$ & $23(10.3)$ & \\
\hline $50-59$ & $80(29.3)$ & $51(22.9)$ & \\
\hline $60-69$ & $100(36.6)$ & $97(43.5)$ & \\
\hline$>70$ & $55(20.2)$ & $52(23.3)$ & \\
\hline Gender & & & $0.323 \dagger$ \\
\hline Male & $213(78.0)$ & $182(81.6)$ & \\
\hline Female & $60(22.0)$ & $41(18.4)$ & \\
\hline Tumor type & & & $0.011 \dagger$ \\
\hline Adenocarcinoma & $224(82.1)$ & $201(90.1)$ & \\
\hline SCC & 49 (17.9) & $22(9.9)$ & \\
\hline \multicolumn{4}{|l|}{ Oncological therapy } \\
\hline Neoadjuvant - all types & $176(66.4)$ & $149(66.8)$ & $0.584 \dagger$ \\
\hline Neoadjuvant chemoRx & $126(71.5)$ & $109(73.1)$ & $0.493 \dagger$ \\
\hline Neoadjuvant CRTx & $50(28.4)$ & $40(26.8)$ & $0.914 \dagger$ \\
\hline Surgery alone & 97 (35.6) & $74(33.2)$ & $0.584 \dagger$ \\
\hline Operation type & & & $<0.0001$ \\
\hline TTO & $150(54.9)$ & $83(37.2)$ & \\
\hline THO & $93(34.1)$ & $107(50.0)$ & \\
\hline 3 Stage & $0(0)$ & $6(2.7)$ & \\
\hline Open and close & $30(11.0)$ & $27(12.1)$ & \\
\hline Pathological T stage & & & $0.012 \dagger$ \\
\hline $\mathrm{HGD} / \mathrm{CPR}$ & $16(5.8)$ & $17(7.6)$ & \\
\hline $\mathrm{T} 1$ & $33(12.1)$ & $48(21.5)$ & \\
\hline $\mathrm{T} 2$ & $31(11.4)$ & $24(10.8)$ & \\
\hline $\mathrm{T} 3$ & $141(51.6)$ & $101(45.3)$ & \\
\hline $\mathrm{T} 4$ & $22(8.1)$ & $6(2.7)$ & \\
\hline Open and close & $30(11.0)$ & $27(12.1)$ & \\
\hline Pathological N stage & & & $0.381 \dagger$ \\
\hline N0 & $112(41.0)$ & $103(46.2)$ & \\
\hline N1 & $67(24.5)$ & $48(21.5)$ & \\
\hline $\mathrm{N} 2$ & $38(13.9)$ & $33(14.8)$ & \\
\hline N3 & $26(9.5)$ & $12(5.4)$ & \\
\hline Open and close & $30(11.0)$ & $27(12.1)$ & \\
\hline Pathological Stage (TNM 7) & & & $0.069 \dagger$ \\
\hline CPR & $15(5.0)$ & $13(5.8)$ & \\
\hline Stage I & $48(17.6)$ & $56(25.1)$ & \\
\hline Stage II & $60(22.0)$ & $48(19.7)$ & \\
\hline Stage III & $20(44.0)$ & $75(33.6)$ & \\
\hline Stage IV & $0(0)$ & $2(0.8)$ & \\
\hline $\mathrm{R} 1$ resection & $83(30.4)$ & $83(37.2)$ & $0.198 \dagger$ \\
\hline Operative mortality & $11(4.0)$ & $6(2.7)$ & $0.415 \dagger$ \\
\hline
\end{tabular}

ACA adenocarcinoma, SCC squamous cell carcinoma, neoadjuvant therapy -all types, chemotherapy/chemo radiotherapy; neoadjuvant chemoRx, neoadjuvant chemotherapy, neoadjuvant CRTx, neoadjuvant chemo radiotherapy, THO trans hiatal esophagectomy, TTO transthoracic esophagectomy; 3 stage 3-stage esophagectomy; $H G D / C P R$ high-grade dysplasia/complete pathological response, $R 1$ positive resection margin, $\S$ Mann-Whitney $U$ test; $\uparrow$ Chi-squared test

\section{Overall survival}

For patients with all pathological stages of disease, median OS was 28 months in the pre-PET/CT cohort, compared with 50 months in the post-PET/CT cohort ( $p=0.004$; Fig. 4). One-, 2-, and 3-year cumulative OS in the pre-PET/CT cohort was 79.1, 55.7, and $42.5 \%$, respectively, compared with $86.2,68.8$, and $57.8 \%$ in the post-PET/CT cohort. Following PET/CT introduction, the median-, 1-, 2-, and 3-year OS increased by 22 months, 7.1, 13.1, and

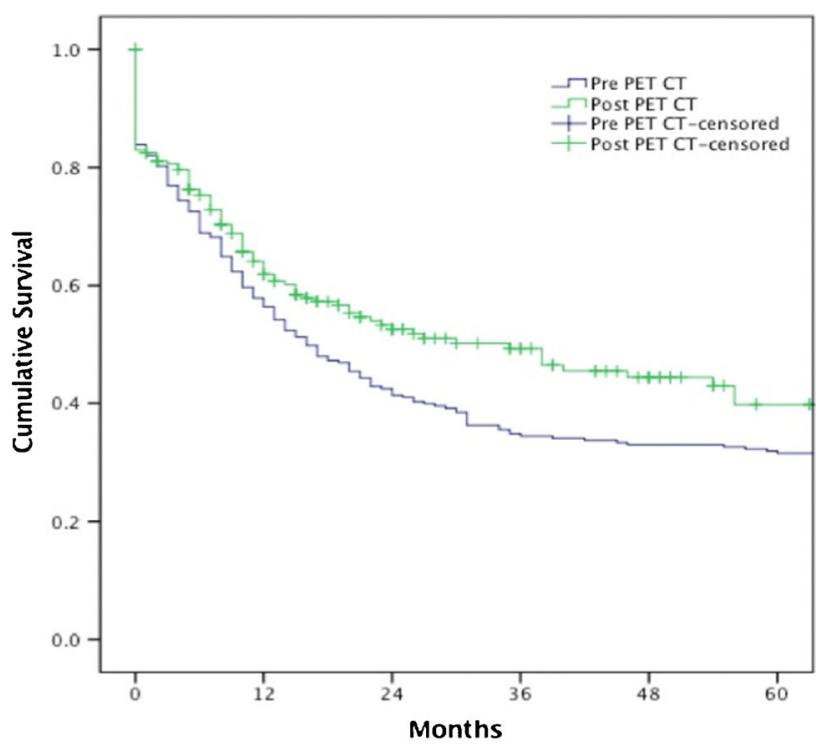

Number at risk

$\begin{array}{llccccc}\text { Time (months) } & 0 & 12 & 24 & 36 & 48 & 60 \\ \text { Pre PET CT } & 273 & 216 & 152 & 116 & 97 & 92 \\ \text { Post PET CT } & 223 & 174 & 113 & 71 & 51 & 29\end{array}$

Log Rank (Mantel-Cox) Chi Squared 3.964 df $1 \quad p=0.046$

Fig. 3 Cumulative disease-free survival related to introduction of PET/ CT

$15.3 \%$, respectively. All factors associated with OS on univariable analysis are shown in Table 3.

Table 2 Univariable analysis of factors associated with disease-free survival

\begin{tabular}{lllc}
\hline & $\mathrm{Chi}^{2}$ & df & $p$ value \\
\hline Gender & 0.446 & 1 & 0.504 \\
Age & 3.427 & 3 & 0.330 \\
Tumor histology & 0.164 & 1 & 0.686 \\
PET/CT & 3.964 & 1 & $\mathbf{0 . 0 4 6}$ \\
PET/CT scanner & 0.244 & 1 & 0.621 \\
Neoadjuvant therapy (all types) & 10.837 & 1 & $\mathbf{0 . 0 0 1}$ \\
Neoadjuvant chemoRx & 19.474 & 1 & $<\mathbf{0 . 0 0 0 1}$ \\
Neoadjuvant CRTx & 0.684 & 1 & 0.408 \\
Surgery type* & 2.620 & 2 & 0.270 \\
pT stage & 390.092 & 5 & $<\mathbf{0 . 0 0 0 1}$ \\
pN stage & 418.258 & 4 & $<\mathbf{0 . 0 0 0 1}$ \\
R1 resection & 365.946 & 2 & $<\mathbf{0 . 0 0 0 1}$ \\
\hline
\end{tabular}

$d f$ degrees of freedom, Age $<50,50-59,60-79,>70$, PET/CT location, Cheltenham/Cardiff, Neoadjuvant therapy -all types, chemotherapy/ chemo radiotherapy, Neoadjuvant chemoRx neoadjuvant chemotherapy, Neoadjuvant CRTX, neoadjuvant chemo radiotherapy, Surgery type *Trans hiatal esophagectomy/trans thoracic esophagectomy/3-stage esophagectomy, $p T$ pathological $\mathrm{T}$ stage, $p N$ pathological $\mathrm{N}$ stage, $R I$ positive resection margin 


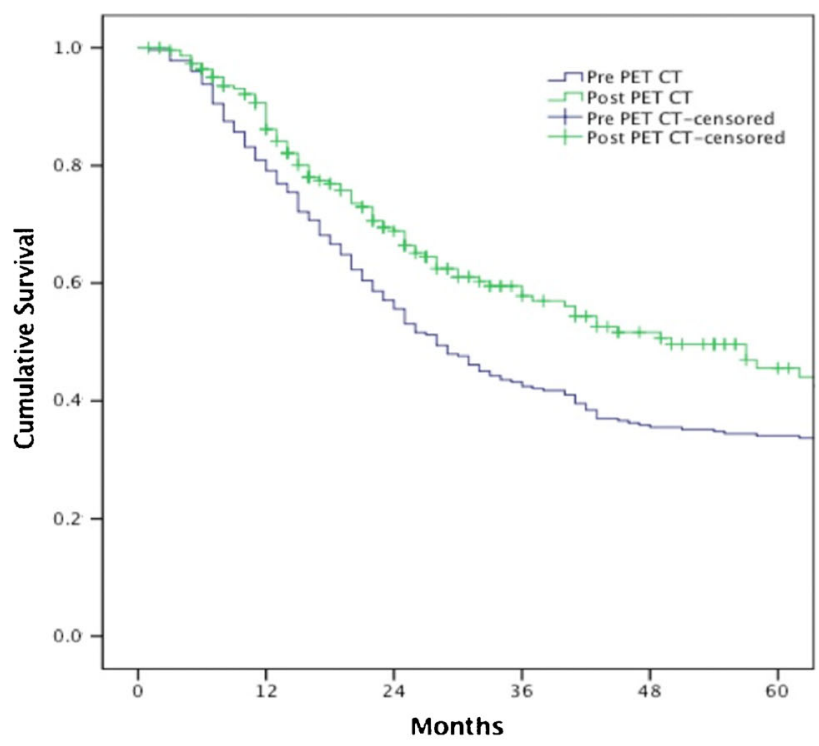

Number at risk

$\begin{array}{llccccc}\text { Time (months) } & 0 & 12 & 24 & 36 & 48 & 60 \\ \text { Pre PET CT } & 273 & 154 & 113 & 94 & 90 & 86 \\ \text { Post PET CT } & 223 & 113 & 74 & 57 & 41 & 23\end{array}$

Log Rank (Mantel-Cox) Chi Squared 8.388 df $1 \quad p=0.004$

Fig. 4 Cumulative overall survival related to introduction of PET/CT

\section{Multivariable analysis}

The factors found to be significantly associated with DFS and OS on univariable analysis were entered into a multivariable analysis using Cox's proportional hazards model, the results of which are shown in Table 4 . The number of events per variable was 38.14 .

\section{Recurrence rates}

Table 5 illustrates the recurrence patterns within the two cohorts. The overall number of patients diagnosed with cancer recurrence was 199 (45.3\%).

Recurrent cancer was observed in 125 patients $(51.4 \%)$ in the pre-PET/CT cohort, compared with 74 patients in the postPET/CT cohort $\left(37.8 \%, \mathrm{Chi}^{2} 8.199\right.$; df $\left.1, p=0.004\right)$. The site of recurrent cancer was less likely to be distant in location after PET/CT introduction (39.5 vs. $27.0 \%, p=0.006$ ).

\section{Discussion}

This is the first study and propensity score analysis to demonstrate a positive significant correlation between the introduction of PET/CT into an EC staging algorithm and a reduction
Table 3 Univariable analysis of factors associated with overall survival

\begin{tabular}{lllc}
\hline & $\mathrm{Chi}^{2}$ & df & p value \\
\hline Gender & 0.246 & 1 & 0.620 \\
Age & 4.609 & 3 & 0.203 \\
Tumor histology & 0.032 & 1 & 0.859 \\
PET/CT & 8.388 & 1 & $\mathbf{0 . 0 0 4}$ \\
PET/CT scanner & 0.306 & 2 & 0.580 \\
Neoadjuvant therapy (all types) & 10.837 & 1 & $\mathbf{0 . 0 0 1}$ \\
Neoadjuvant chemoRx & 17.388 & 1 & $<\mathbf{0 . 0 0 0 1}$ \\
Neoadjuvant CRTx & 1.028 & 1 & 0.311 \\
Surgery type* & 4.356 & 2 & 0.113 \\
pT stage & 160.877 & 5 & $<\mathbf{0 . 0 0 0 1}$ \\
pN stage & 177.154 & 4 & $<\mathbf{0 . 0 0 0 1}$ \\
R1 & 136.000 & 2 & $<\mathbf{0 . 0 0 0 1}$ \\
\hline
\end{tabular}

$d f$ degrees of freedom, Age $<50,50-59,60-79,>70$, PET/CT location, Cheltenham/Cardiff, Neoadjuvant therapy -all types, chemotherapy/ chemo radiotherapy, Neoadjuvant chemoRx neoadjuvant chemotherapy, Neoadjuvant CRTX, neoadjuvant chemo radiotherapy, Surgery type *Trans hiatal esophagectomy/trans thoracic esophagectomy/3-stage esophagectomy, $p T$ pathological $\mathrm{T}$ stage, $p N$ pathological $\mathrm{N}$ stage, $R 1$ positive resection margin

in cancer recurrence, with a commensurate increase in durations of survival, irrespective of stage, in patients undergoing potentially curative surgery.

In keeping with previous reports [5, 6], PET/CT upstaged 78 patients $(13.2 \%)$, changing their treatment modality and precluding surgery. Median-, 1-, and 3-year OS increased significantly by 22 months, 13.1 and $15.3 \%$, respectively after introduction of PET/CT. Cancer recurrence was $13.6 \%$ less common after PET/CT introduction, with fewer locoregional and distant recurrence events when compared with historical controls. Consequently, the hypothesis addressed, namely that introduction of PET/CT into the routine EC staging algorithm was associated with improved OS after potentially curative surgery, was proven correct.

Reports regarding the influence of PET/CT within EC staging algorithms on patient long-term outcomes related to

Table 4 Multivariable analysis of factors associated with disease-free and overall survival

\begin{tabular}{lllc}
\hline Variable & HR & $95 \%$ CI & $p$ value \\
\hline $\begin{array}{l}\text { Disease-free survival } \\
\text { pT stage }\end{array}$ & 1.526 & $1.31-1.78$ & $<0.0001$ \\
pN stage & 1.371 & $1.26-1.49$ & $<0.0001$ \\
Overall survival & & & \\
PET/CT introduction & 0.688 & $0.53-0.89$ & 0.004 \\
pT stage & 1.496 & $1.28-1.75$ & $<0.0001$ \\
pN stage & 1.114 & $1.04-1.19$ & 0.001 \\
\hline
\end{tabular}

$p T$ pathological T stage, $p N$ pathological $\mathrm{N}$ stage 
Table 5 Recurrence rates related to cohort for patients who received curative surgery

\begin{tabular}{lllll}
\hline & $\begin{array}{l}\text { Pre-PET/ } \\
\text { CT }\end{array}$ & $\begin{array}{l}\text { Post-PET/ } \\
\text { CT }\end{array}$ & Total & $p$ value \\
\hline Number of patients* & 243 & 196 & 439 & \\
Recurrence & $125(51.4)$ & $74(37.8)$ & 199 & $\mathbf{0 . 0 0 4}$ \\
Site of recurrence & & & & \\
Locoregional & $55(22.6)$ & $34(17.3)$ & 89 & 0.171 \\
$\quad$ Distant & $96(39.5)$ & $53(27.0)$ & 149 & $\mathbf{0 . 0 0 6}$ \\
Time to recurrence (months) & & & & 0.308 \\
Overall & $15(2-85)$ & $10(2-93)$ & & 0.392 \\
Locoregional & $15(2-85)$ & $14.5(4-93)$ & & 0.7 \\
Distant & $14(2-72)$ & $10(2-69)$ & 57 & \\
Open and close laparotomy & 30 & 27 & & \\
\hline
\end{tabular}

Figures are numbers of patients with percentages in parentheses; Site of recurrence, inclusive of patients diagnoses with both locoregional and distant disease at time of diagnosis;

*Excluding open and close procedures recurrence and durations of survival are few. Torrance et al. [8], from Cheltenham, England, reported in a retrospective review of $200 \mathrm{EC}$ patients undergoing $\mathrm{PET} / \mathrm{CT}, 128$ of who underwent esophageal resection. Although PET/CT altered treatment intent in 19 patients $(9.5 \%)$, no significant difference was noted in post-operative mortality, or early recurrence where PET/CT was performed when adjusted for age, gender, stage, or neoadjuvant chemotherapy (odds ratio $1.136, p=$ $0.761)$. Moreover, PET/CT had no significant effect on survival $\left(\mathrm{Chi}^{2} 0.710, p=0.400\right)$. The difference in survival preand post-PET/CT was approximately 2,6 , and $7 \%$ at 1,2 , and 3 years, compared with $7.1,13.1$, and $15.3 \%$ in this study. Torrance et al. concluded that PET/CT improved the accuracy of EC staging, avoiding potentially unnecessary surgery, and contended that missed occult metastases did not appear to be the primary cause of early EC recurrence.

The current study has potential inherent limitations. The PET/CT examinations were performed at two centers, using different scanners, protocols, and uptake times, with patients in the early part of the study referred to site 1 (Philips 16 slice Gemini GXL), prior to the opening of site 2's PET/CT in 2010. The main differences between the two centers were firstly, a 60-min uptake time on the first scanner (site 1), and a 90-min uptake time in site 2. Longer uptake times lead to higher tumor-to-background tracer uptake and may therefore increase conspicuity of nodal and distant metastases [23]. Secondly, the site 2 scanner had time of flight correction whereas the site 1 scanner did not. Time-of-flight reconstructions improve signal-to-noise ratio and improve lesion conspicuity [24]. Thirdly, at the site 1 center, images were acquired for $4 \mathrm{~min}$ per bed position, whereas at site 2, the acquisition was $3 \mathrm{~min}$ per bed position. This would be expected to lead to some improvement of image quality at the site 1 center, provided that the patient was able to remain motionless throughout the longer acquisition, which might mitigate the other factors. The potential heterogeneity introduced within the PET/CT imaged group was a reason why the location of the $\mathrm{PET} / \mathrm{CT}$ scan was a covariate within the propensity score and as a result, adds great strength to this study.

The study included patients treated over a 17 -year period with a variety of treatment strategies. Evolution in UGI cancer practice would have naturally occurred during the time frame of this study, including neoadjuvant therapy regimen modifications, more detailed patient risk profile assessment related to patients' fitness for surgery including objective assessment of physical fitness with cardiopulmonary exercise testing, centralization of surgical services, and the introduction of an Enhanced Recovery Program, all of which might represent potential confounding factors.

Conversely, the strengths of the study are that the data was collected prospectively, from a well-defined geographical area served by an established regional UGI cancer network and multidisciplinary team. This team included six experienced specialist surgeons, with a referring population base of 1.8 million, accepting over 500 cases per year, generating in excess of 100 potentially curative esophagogastric resections, whose outcome data is well audited and of public record [25]. The survival and prognostic data are especially robust because no patients were lost to follow-up and causes and dates of death were obtained from death certificates provided by the Office for National Statistics. Moreover, the improvement in survival cannot be explained by poor outcomes in the historical control cohort, which compare favorably with the clinical outcomes data published in the most recent NOGCA report [26]. NOGCA cumulative survival at 1, 2, and 3 years was approximately 70, 50, and $40 \%$ compared with $79.1,55.7$, and $42.5 \%$ in propensity matched historical controls. Furthermore, NOGCA reported 30-day mortality of 4.5\%, between 2007 and 2009, comparable with the $4 \%$ observed in the propensity matched historical cohort of this study. 
Multivariable model analyses have been considered the orthodox and preferred statistical way of assessing the effect of possible predictors on outcomes after controlling for baseline characteristics, yet their suitability depends on consistent assumptions underlying any given model. Regression analyses of DFS and OS by means of propensity scores overcome some potential biases, and although it is considered the most useful statistical method for controlling confounders, providing appropriate estimates even when faced with extreme correlation between confounders and the exposure [27], it cannot account for unknown confounders [28]. Propensity score analysis is well suited when several risk-adjusted outcomes are under assessment (DFS and OS), because such scoring simplifies multiple outcome weighting, which once calculated can be related to each specific outcome measure.

In conclusion, this propensity score regression analysis further supports the use of PET/CT in EC staging pathways, and in the absence of an adequately powered randomized controlled trial, provides the best level of research evidence regarding the influence of PET/CT on outcome after potentially curative esophagectomy for cancer. Risk profile assessment represents an important development in the selection algorithm for patients diagnosed with invasive EC. While this is often assumed to relate to patients' physical fitness, clearly avoiding hopeless radical, and unnecessary surgery, in patients with undetected occult metastases is an important allied strategy, if EC treatment outcomes are to be optimized. The findings of this study have shown that the introduction of PET/CT into the global patient assessment process changed the risk profile of over one in ten patients, reduced global recurrence by one-quarter, and improved median survival by a full year. A health economic analysis of the cost-effectiveness of PET/CT is now desirable to estimate the potential allied clinical and fiscal benefits available to upper gastrointestinal cancer networks.

Acknowledgements The authors thank statistician Dr Mark Kelsen, for ensuring correct use of statistical methodologies. The authors also acknowledge the following members of the South East Wales Regional Cancer Network for their contribution: Consultant surgeons GWB Clark, GRJC Blackshaw, TJ Havard, X Escofet and M Henwood.

Funding No funding was received for this research.

\section{Compliance with ethical standards}

Conflict of interest Mr. Neil Patel declares that he has no conflict of interest.

Dr. K Foley declares that he has no conflict of interest.

Mr. A Powell declares that he has no conflict of interest.

Miss J Wheat declares that she has no conflict of interest.

Mr. D Chan declares that he has no conflict of interest.

Dr. P Fielding declares that he has no conflict of interest.

Dr. A Roberts declares that he has no conflict of interest.

Prof. W Lewis declares that he has no conflict of interest.

No animals were involved in this research.
Ethical approval All procedures performed in studies involving human participants were in accordance with the ethical standards of the institutional research committee and with the 1964 Helsinki Declaration and its later amendments or comparable ethical standards.

Informed consent Participants in this study were not required by the Institutional Review Board to provide written consent for this study because this work was deemed an evaluation of clinical service.

Open Access This article is distributed under the terms of the Creative Commons Attribution 4.0 International License (http:// creativecommons.org/licenses/by/4.0/), which permits unrestricted use, distribution, and reproduction in any medium, provided you give appropriate credit to the original author(s) and the source, provide a link to the Creative Commons license, and indicate if changes were made.

\section{References}

1. Schmidt T, Lordick F, Herrmann K, Ott K. Value of functional imaging by PET in esophageal cancer. J Natl Compr Cancer Netw. 2015;13:239-47.

2. Flamen P, Lerut A, Van Cutsem E, De Wever W, Peeters M, Stroobants S, et al. Utility of positron emission tomography for the staging of patients with potentially operable esophageal carcinoma. J Clin Oncol. 2000;18:3202-10.

3. van Westreenen HL, Westerterp M, Bossuyt PM, Pruim J, Sloof GW, van Lanschot JJ, et al. Systematic review of the staging performance of $18 \mathrm{~F}$-fluorodeoxyglucose positron emission tomography in esophageal cancer. J Clin Oncol. 2004;22:3805-12.

4. Allum WH, Blazeby JM, Griffin SM, Cunningham D, Jankowski JA, Wong R. Guidelines for the management of oesophageal and gastric cancer. Gut. 2011;60:1449-72.

5. Noble F, Bailey D, Tung K, Byrne JP. Impact of integrated PET/CT in the staging of oesophageal cancer: a UK population-based cohort study. Clin Radiol. 2009;64:699-705.

6. Blencowe NS, Whistance RN, Strong S, Hotton EJ, Ganesh S, Roach $\mathrm{H}$, et al. Evaluating the role of fluorodeoxyglucose positron emission tomography-computed tomography in multi-disciplinary team recommendations for oesophago-gastric cancer. $\mathrm{Br} \mathrm{J}$ Cancer. 2013;109:1445-50.

7. Kelly S, Harris KM, Berry E, Hutton J, Roderick P, Cullingworth J, et al. A systematic review of the staging performance of endoscopic ultrasound in gastro-oesophageal carcinoma. Gut. 2001;49:534-9.

8. Torrance AD, Almond LM, Fry J, Wadley MS, Lyburn ID. Has integrated $18 \mathrm{~F}$ FDG PET/CT improved staging, reduced early recurrence or increased survival in oesophageal cancer? Surgeon. 2015;13:19-33.

9. Royal College of Surgeons Clinical Effectiveness Unit. National Oesophago-Gastric Cancer Audit 2008. London, UK; 2008.

10. Rosenbaum PR, Rubin DB. The central role of the propensity score in observational studies for causal effects. Biometrika. 1983;70:4155 .

11. Austin PC. An introduction to propensity score methods for reducing the effects of confounding in observational studies. Multivar Behav Res. 2011;46:399-424.

12. D'Agostino RB Jr. Propensity score methods for bias reduction in the comparison of a treatment to a non-randomized control group. Stat Med. 1998;17:2265-81.

13. Karran A, Blake P, Chan D, Reid TD, Davies IL, Kelly M, et al. Propensity score analysis of oesophageal cancer treatment with surgery or definitive chemoradiotherapy. Br J Surg. 2014;101:502-10. 
14. Karran A, Wheat J, Chan D, Blake P, Barlow R, Lewis WG. Propensity score analysis of an enhanced recovery programme in upper gastrointestinal cancer surgery. World J Surg. 2016;40:164554.

15. Sobin LH, Gospodarowicz MK, Wittekind CH. UICC TNM classification of malignant tumours. 7th ed. New York: Wiley; 2009.

16. Chan DS, Fielding P, Roberts SA, Reid TD, Ellis-Owen R, Lewis WG. Prognostic significance of 18-FDG PET/CT and EUS-defined tumour characteristics in patients with oesophageal cancer. Clin Radiol. 2013;68:352-7.

17. Crosby TD, Brewster AE, Borley A, Perschky L, Kehagioglou P, Court J, et al. Definitive chemoradiation in patients with inoperable oesophageal carcinoma. Br J Cancer. 2004;90:70-5.

18. Stephens MR, Lewis WG, Brewster AE, Lord I, Blackshaw GR, Hodzovic I, et al. Multidisciplinary team management is associated with improved outcomes after surgery for esophageal cancer. Dis Esophagus. 2006;19:164-71.

19. Gwynne S, Hurt C, Evans M, Holden C, Vout L, Crosby T. Definitive chemoradiation for oesophageal cancer-a standard of care in patients with non-metastatic oesophageal cancer. Clin Oncol (R Coll Radiol). 2011;23:182-8.

20. Mapstone N. Dataset for the histopathological reporting of oesophageal carcinoma. 2nd ed. London: Royal College of Pathologists; 2007.

21. Kelsen DP, Ginsberg R, Pajak TF, Sheahan DG, Gunderson L, Mortimer J, et al. Chemotherapy followed by surgery compared with surgery alone for localized esophageal cancer. N Engl J Med. 1998;339:1979-84.

22. Medical Research Council Oesophageal Cancer Working Group. Surgical resection with or without preoperative chemotherapy in oesophageal cancer: a randomised controlled trial. Lancet. 2002;359:1727-33.

23. Chirindel A, Alluri KC, Tahari AK, Chaudhry M, Wahl RL, Lodge MA, et al. Liver standardized uptake value corrected for lean body mass at FDG PET/CT: effect of FDG uptake time. Clin Nucl Med. 2015;40:e17-22.

24. Surti S. Update on time-of-flight PET imaging. J Nucl Med. 2015;56:98-105.

25. Morgan MA, Lewis WG, Casbard A, Roberts SA, Adams R, Clark GW, et al. Stage-for-stage comparison of definitive chemoradiotherapy, surgery alone and neoadjuvant chemotherapy for oesophageal carcinoma. Br J Surg. 2009;96:1300-7.

26. National Oesophago-Gastric Cancer Audit. An Audit of the Care Received by People with Oesophago-Gastric Cancer in England and Wales Annual Report.; 2016.

27. Cook EF, Goldman L. Performance of tests of significance based on stratification by a multivariate confounder score or by a propensity score. J Clin Epidemiol. 1989;42:317-24.

28. Nicholas J, Gulliford MC. Commentary: what is a propensity score? Br J Gen Pract. 2008;58:687. 